\section{P5.102 ANTIMICROBIAL SUSCEPTIBILITY OF NEISSERIA GONORRHOEAE IN NANJING, CHINA, 2011-2012}

doi:10.1136/sextrans-2013-051184.1146

${ }^{1} \mathbf{X}$ Su, ${ }^{1} W$ Le, ${ }^{1} S$ Li, ${ }^{1 B}$ Wang, ${ }^{2 P}$ Rice. 'National Center for STD Control, Institute of Dermatology,CAMS, Nanjing, China; 'University of Massachusetts Medical School, Worcester, MA, United States

Background A study of gonococcal transmission from men to their female contacts has been under investigation at the STD clinic in Nanjing, China since April 2011. Men with symptomatic urethral discharge presenting for diagnosis and management have been screened for gonococcal infection and Neisseria gonorrhoeae strains isolated. Monitoring antimicrobial resistance (AMR) of N. gonorrhoeae is crucial for guiding effective treatment. Here, we report the results of amtimicrobial susceptibility testing of these gonococcal strains between April 2011 and 2012.

Methods We determined minimum inhibitory concentrations (MICs) for 334 concecutively obtained gonococcal isolates by agar dilution testing for: penicillin, tetracycline, ciprofloxacin, spectinomycin and ceftriaxone. $\beta$-lactamase production was determined by paper acidometric testing.

Results The prevalence of PPNG and TRNG was 36.53\% (122/334) and $38.32 \%$ (128/334), respectively. Chromosomally-mediated resistance to penicillin and tetracycline was $87.26 \%(185 / 212)$ and $99.03 \%(204 / 206)$, respectively. Chromosomal resistance to ciprofloxacin (ORNG) was detected in $98.80 \%(330 / 334)$ of the isolates. All gonococcal isolates were susceptible to spectinomycin. Gonococcal isolates having ceftriaxone MICs $\geq 0.06 \mathrm{mg} / \mathrm{L}$ accounted for $27.54 \%$ (92/334); among them two isolates with MICs of $0.25 \mathrm{mg} / \mathrm{L}$ and 13 having MICs of $0.125 \mathrm{mg} / \mathrm{L}$.

Conclusion Among N. gonorrhoeae strains isolated in Nanjing, chromosomally-mediated resistance to penicillin, tetracycline and ciprofloxacin was high. Ceftriaxone and spectinomycin should be considered effective anti-microbial agents for the treatment of gonorrhoea in Nanjing, but and susceptibility of $N$. gonorrhoeae to these antimicrobial agents should be monitored continuously.

\section{P5.103 INCREASE OF AFRICAN-TYPE PENICILLINASE-PRODUCING N. GONORRHOEAE STRAINS ISOLATED IN GUANGZHOU, CHINA, 2001-2011}

doi:10.1136/sextrans-2013-051184.1147

H Zheng, B Yang, X Wu, X Qin, J Huang, Y Xue, W Zeng, J Ou, Y Lan, S Tang. Guangdong Provincial Center for Skin Diseases and STIs Control, Guangzhou, China

Background The continuing spread of drug-resistant gonococci has posed a challenge for successful treatment worldwide. Guangdong Province in South China has one of the highest resistance rates of gonococci in China and a large number of international migrants. We investigated the in vitro antimicrobial susceptibility and genotypes of N. gonorrhoeae isolated in Guangzhou, the capital city, in 2001-11.

Methods MICs to penicillin, ceftriaxone, tetracycline, ciprofloxacin, and spectinomycin were determined by agar plate dilution and susceptibilities were interpreted according to WHO standards. Penicillinase-producing $\mathrm{N}$. gonorrhoeae (PPNG) was determined by paper acidometric testing and high-level tetracycline resistant $\mathrm{N}$. gonorrhoeae (TRNG) by MIC. The isolates with resistant plasmids were genotyped by PCR

Results Of 1250 consecutive gonococci isolated during 2001-11, no ceftriaxone and spectinomycin resistant strains were found, but the prevalence of less susceptible strains to ceftriaxone rose from $17 \%$ to $46.5 \%$. The $\mathrm{MIC}_{90}$ for ceftriaxone showed intermediate sensitivity $(0.06-0.125 \mu \mathrm{g} / \mathrm{mL})$ and spectinomycin near the resistant level $(16-$ $32 \mu \mathrm{g} / \mathrm{mL}$ ). The resistance to penicillin, tetracycline and ciprofloxacin were from $85 \%, 85 \%$ and $78 \%$ in 2001 to $81.9 \%, 100 \%$ and $98.4 \%$ in 2011, respectively. Of 349 (27.9\%) PPNG and 539 (43.1\%) TRNG detected. $232(18.6 \%)$ strains were both PPNG and TRNG. PPNG increased from $17.0 \%$ to $32.3 \%$ and TRNG from $26.0 \%$ to $41.7 \%$ during 2001-10. Genotyping TEM-1 gene showed 94.8\% PPNGs carrying the Asia-type $\beta$-lactamase plasmids. The Africa-type PPNG (1, $1.3 \%$ ) emerged in 2008 and has increased to 8(6.2\%) in 2011. Genotyping of tetM gene showed all TRNGs were Dutch variants.

Conclusion Gonorrhea resistance continues to be a major public health problem in Guangzhou. The emergence and increase of African-type PPNG may be related to the large African diasporas in Guangzhou, migration of Chinese to Africa, or other migration patterns. More research is needed to determine what practises, systems and behaviours contribute to escalating resistance patterns.

\section{P5.104 PRELIMINARY STUDY OF THE SENSITIVITY OF NEISSERIA GONORRHOEAE TO ANTIBIOTICS IN STRAINS ISOLATED FROM PEOPLE WITH RISKY SEXUAL BEHAVIOUR IN THE CZECH REPUBLIC}

doi:10.1136/sextrans-2013-051184.1148

'P Prochazka, ${ }^{2} \mathbf{D}$ Vanousova, ${ }^{3} \mathrm{~V}$ Vanis, ${ }^{3} \mathrm{~V}$ Jindrak, ${ }^{3} \mathrm{~J}$ Kubele, ${ }^{3} \mathrm{Z}$ Zemanova, ${ }^{4} \mathrm{P}$ Dryak, ${ }^{4} \mathrm{~B}$ Sykorova, ${ }^{5} \mathrm{H}$ Zakoucka, ${ }^{2} \mathrm{~J}$ Hercogova. ${ }^{1}$ Department of Venereology, Medicentrum Beroun, Prague, Czech Republic; 'Department of Dermatovenereology; 2nd Faculty of Medicine, Charles University and Na Bulovce Hospital, Prague, Czech Republic; ${ }^{3}$ Department of Microbiology, Na Homolce Hospital, Prague, Czech Republic; ${ }^{4}$ Department of Microbiology, Na Bulovce Hospital, Prague, Czech Republic; ${ }^{5}$ National Reference Laboratory for Syphilis, National Institute for Public Health, Prague, Czech Republic

Background Neisseria gonorrhoeae infection is currently a serious medical problem for widespread of multi-drug resistant strains causing difficult to treat illnesses. Resistance to fluoroquinolones, azithromycin as well as recently reported cases of resistance to cefixime and ceftriaxone represents important health threat.

Methods The first consecutive isolates of Neisseria gonorrhoeae obtained from patients with risky sexual behaviour (promiscuity, commercial sex, gay community including HIV infected individuals) suspected of infection or carriage, coming to two dermatovenereology clinics in Prague from June 2012 to February 2013, were included Strains were detected from clinical samples (urethral, vaginal/cervical, rectal or throat swabs) using standard culture technique. Susceptibility to fluoroquinolones and macrolides was routinely determined using disc diffusion test. E-test technique was done to establish MIC values of penicillin and cefixime. Clinical and epidemiological characteristics of patients and strains were studied using standardised protocol.

Results A total of $82 \mathrm{~N}$. gonorrhoeae consecutive isolates were tested. The resistance rate to ciprofloxacin was $40 \%$, resistance to erythromycin was $3.8 \%$, respectively. Penicillin MIC equal or higher than $1.0 \mathrm{~g} / \mathrm{l}$ was found in $21 \%$ of strains. Cefixime MIC values were equal to $0.125 \mathrm{~g} / \mathrm{l}$ in $4.9 \%$ of strains, no strain with MIC higher than $0.125 \mathrm{~g} / 1$ was identified. The patients were treated with azithromycin, doxycycline, cefixime, ceftriaxone, penicillin or combinations of antibiotics. Treatment failure was observed in 7 cases. Association of treatment failure with antimicrobial resistance should be clarified via following clinical studies.

Conclusions Preliminary results confirmed important occurrence of resistance of $\mathrm{N}$. gonorrhoeae to essential antimicrobials in high risk population in the Czech Republic. Similarly to other European countries, increasing MIC values of penicillin and cefixime indicate growing risk of difficult to treat $\mathrm{N}$. gonorrhoeae infections.

\section{P5.105 IDENTIFICATION AND FUNCTIONAL ANNOTATION OF SECRETED PROTEINS OF CHLAMYDIA TRACHOMATIS USING BIOINFORMATICS TOOLS}

doi:10.1136/sextrans-2013-051184.1149

'P K Mishra, 'S C Sonkar, ' $\mathrm{S}$ R Raj, ${ }^{2} \mathrm{U}$ Chaudhry, 'D Saluja. 'Dr. B. R. Ambedkar Center for Biomedical Research, University of Delhi, Delhi India, Delhi, India; ${ }^{2}$ Bhaskaracharya College of Applied Sciences, University of Delhi, Delhi, India 